\title{
Non-Conventional Network Analysis
}

\author{
Dr. Péter Kádár, senior member of IEEE \\ Óbuda University \\ Dept. of Power Systems, Alternative Energy Technologies Knowledge Centre \\ Bécsi u. 94. Budapest H-1034 HUNGARY \\ Phone: +36 209447 241; fax: +30 1250 0940; e-mail: kadar.peter@kvk.uni-obuda.hu
}

\begin{abstract}
The development, operational control and evaluation of the events in the power system necessitate different network calculation methods. A stationary grid model is used for load distribution and contingency analysis. The classic load-flow algorithm is an iterative solution for equation system of thousands of variables. For the efficient computation of networks containing huge number of nodes and branches some acceleration and simplification algorithms are used, too (decoupled and DC load-flow).

The emerging structures as the large non-meshed radial networks, microgrids, power quality islands open new frontiers instead of the exhaustive number-crunching techniques. The tasks can be rephrased, and the application of several intelligent computation methods can be relevant, as the artificial neural networks and some optimization techniques. This paper introduces the on- and off-line tasks of the network calculations, the existing methods and the novel techniques.
\end{abstract}

\section{Key words}

Power system, Network calculation, Load-Flow, Metaheuristics, Optimization

\section{The Power Network}

The most important elements of the power system are the Generator, Transformer and the Power lines. These are connected in substations by switching devices. In the latest models the CHP unit (cogeneration heat and power), Electricity storage, Heat storage and consumers, Renewable sources appear with complicated characteristics. Although the number of the element types is low the number of the real elements is really high (see table I.).

We meet new challenges in the network analysis by the Emerging structures as

- Microgrids

- Smart grids

- Power quality islands
- DC systems

- Off-road systems

- Distributed generation, etc.

Table I. - Approximate number of elements in the Hungarian power system

\begin{tabular}{|l|c|c|c|}
\hline & $\begin{array}{l}\text { on high } \\
\text { voltage } \\
\text { level }\end{array}$ & $\begin{array}{l}\text { medium } \\
\text { voltage } \\
\text { level }\end{array}$ & $\begin{array}{l}\text { low } \\
\text { voltage } \\
\text { level }\end{array}$ \\
\hline Generators & 50 & 1000 & 20000 \\
\hline Transformers & 150 & 600 & 50000 \\
\hline Power lines & 500 & 1500 & $\mathrm{x}$ \\
\hline Substations & 30 & 300 & 50000 \\
\hline CHPs & 5 & 300 & $\mathrm{x}$ \\
\hline Electricity storage & 0 & 10 & 10000 \\
\hline Heat storage & 0 & 5 & 1000000 \\
\hline Load (demand) & 200 & 20000 & 7000000 \\
\hline
\end{tabular}

The Hungarian power system's peak load is $7 \mathrm{GW}$, meanwhile the European (ENTSO-E) power system's peak load is $600 \mathrm{GW}$ !

\section{The Network Analysis}

\section{A. The „classic” Load Flow}

The basic tool for the power system calculation is the Load Flow calculation. This calculation method spread with the mainframe computers at the end of the sixties for meshed network calculation with hundreds of nodes and branches. The roles of the Load Flow are

- Network monitoring (see fig.1.)

- Switching simulation

- Overload warning

- Dispatcher training simulator

- Contingency analysis

- Optimal dispatch, etc. 


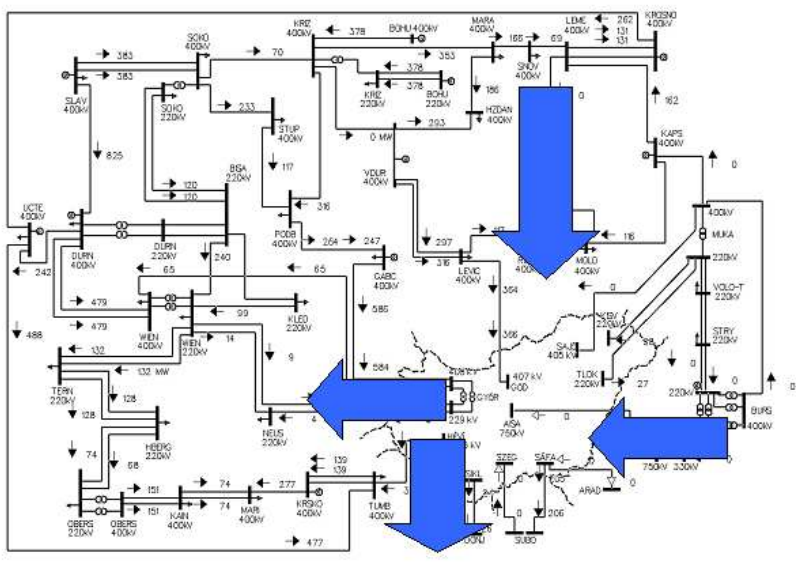

Fig. 1. Investigation of power transit by load-flow

Basic „question” of the Load Flow calculation:

How can a given load demand be satisfied in a stationary manner by a set of sources with static network parameters?

Although in the traditional electrical network calculation all the component parameters are known (R, L, C, sources), the power network dispatcher doesn't handle the power plants and loads as an 'Impedance'. These are known as power source and consumption $(\mathrm{P}, \mathrm{Q})$.

Given data (see fig.2.):

- $\quad$ Load $\left(\mathrm{P}_{\text {load }}, \mathrm{Q}_{\text {load }}\right)$ - consumptions

- Generation possibilities (P, Q, and $\mathrm{S}_{\min , \max }$ ) power plants

- $\quad$ Network parameters (R, X, C, f)

- $\quad$ Limits $\left(\mathrm{S}_{\max }, \mathrm{U}_{\max }, \mathrm{U}_{\min }, \delta_{\max }\right)$

- $\quad$ Topology (network connection + actual switch states)

- $\left(\mathrm{U}_{\text {nodes }}\right.$ initial $)$

Looking for:

- $\quad$ Node voltages (U)

- $\quad$ Flows through the branches $(\mathrm{P}, \mathrm{Q})$

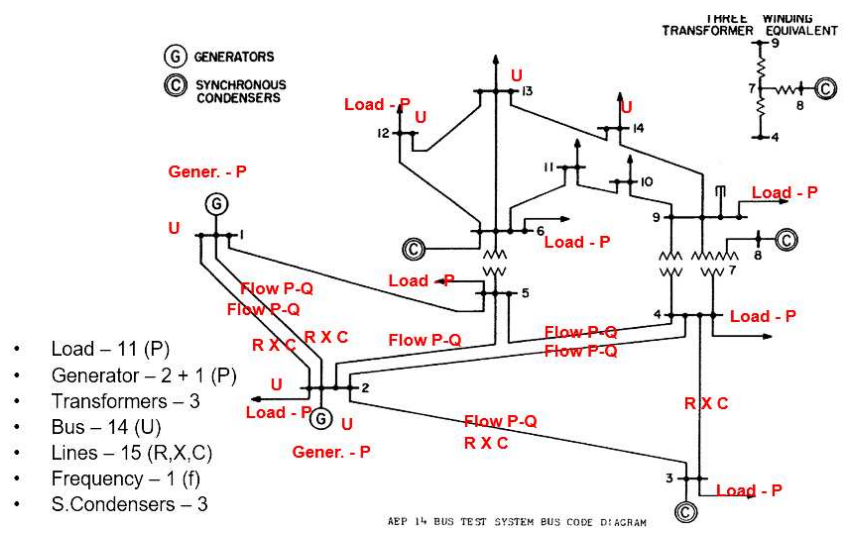

Fig. 2. Input data of the load-flow calculation on AEP 14 bus [1] system

In the classic network there is no direct possibility to interfere in the flow. It evolves just like the water moves through a complicated meshed pipe system. However, we can precisely calculate the happenings. Nowadays through certain FACT (flexible AC Transmission) devices and by special winding transformer the flow can really be adjusted.

\section{A. Traditional LF equations}

$$
\mathbf{I}=\mathbf{Y} \mathbf{U} \quad(\mathrm{I}=\mathrm{U} / \mathrm{R})
$$

Complex, nonlinear equation system, where

$$
\begin{aligned}
& \text { I } \quad \text { vector of branch flows (complex) } \\
& \text { Y admittance matrices of the net } \\
& \text { (complex) } \\
& \text { U vector of node voltages (complex) }
\end{aligned}
$$

The nonlinearity is caused by

- Nonlinear elements

- Voltage dependency of loads

- Frequency dependency of loads

Power is known but the current isn't...

$$
\begin{gathered}
I=\frac{(P-j Q)}{|U|} e^{+j \delta} \quad I=\frac{P}{U} \\
\frac{P_{1}}{U_{1}}=\left(Y_{12}+Y_{13}+Y_{10}\right) U_{1}-Y_{12} U_{2}-Y_{13} U_{3} \\
\frac{P_{2}}{U_{2}}=-Y_{12} U_{1}+\left(Y_{12}+Y_{23}+Y_{20}\right) U_{2}-Y_{23} U_{3} \\
\frac{P_{3}}{U_{3}}=-Y_{13} U_{1}-Y_{23} U_{2}+\left(Y_{13}+Y_{23}+Y_{30}\right) U_{3} \\
U_{1}=\frac{1}{Y_{11}}\left(\frac{P_{1}}{U_{1}}+Y_{12} U_{2}+Y_{13} U_{3}\right) \\
U_{2}=\frac{1}{Y_{22}}\left(\frac{P_{2}}{U_{2}}+Y_{12} U_{1}+Y_{23} U_{3}\right) \\
U_{3}=\frac{1}{Y_{33}}\left(\frac{P_{3}}{U_{3}}+Y_{13} U_{1}+Y_{23} U_{2}\right)
\end{gathered}
$$

An iterative solution is necessary for the not known voltages and nonlinearities. Quick, simplified methods are the decoupled LF with unbundling $(\mathrm{P}+\mathrm{Q})$ or the DC LF method.

\section{B. Branch model}

The load-flow has similar, four pole branch models for all the elements (see fig.3.):

$$
\begin{array}{ll}
\text { - } & \text { line } \\
\text { - } & \text { transformer } \\
\text { - } & \text { (generator) }
\end{array}
$$

The substations are Nodes with zero impedance. We have two equations per branch, but 2 thousand for 1 thousand branches: 


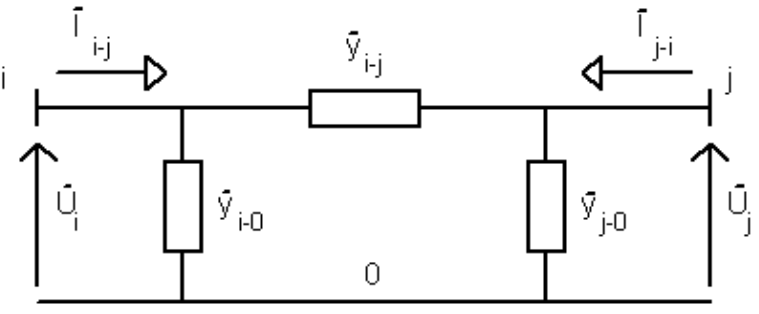

$$
\begin{aligned}
& P_{i}=G U_{i}^{2}+\sum_{j \neq i} B_{i j} U_{i} U_{j} \sin \left(\delta_{i}-\delta_{j}\right)+\sum_{j \neq i} G_{i j} U_{i} U_{j} \cos \left(\delta_{i}-\delta_{j}\right) \\
& Q_{i}=-B_{j} U_{i}^{2}-\sum_{j \neq i} B_{i j} U_{i} U_{j} \cos \left(\delta_{i}-\delta_{j}\right)+\sum_{j \neq i} G_{i j} U_{i} U_{j} \sin \left(\delta_{i}-\delta_{j}\right)
\end{aligned}
$$

Fig. 3. The four-pole model of the branch

The huge equation system can be solved by

- Gauss iteration

- Gauss-Seidel iteration

- Newton-Rhapson iteration, etc.

In the early computer age, it wasn't easy to work with sparse matrices, to invert them.

\section{Off-line and online NA functions}

WHY and WHAT to calculate in the power system? The power system design, the operation requires a model about the behavior of the power system. The No.1. power system model is the load flow that helps us control the currents and voltages.

The Load-flow has a stationary view, other models belong to the short term switching transients or to the wandering waves. Most of the Energy Management System functions contain Load-Flow calculation (real time / off line Network Analysis) - See Table II.

Table II. - EMS functions

\begin{tabular}{|l|l|c|}
\hline function & & $\begin{array}{c}\text { contains } \\
\text { LF }\end{array}$ \\
\hline AGC & automatic generation control & $*$ \\
\hline LF & load-flow (power flow) & $*$ \\
\hline OPF & optimal power flow & $*$ \\
\hline CA/SA & $\begin{array}{l}\text { contingency analysis / } \\
\text { security analysis }\end{array}$ & $*$ \\
\hline MU & $\begin{array}{l}\text { model update - topology } \\
\text { update }\end{array}$ & \\
\hline SE & state estimation & $*$ \\
\hline SC & short circuit calculation & $*$ \\
\hline VS & voltage scheduler & $*$ \\
\hline AVC & automatic voltage control & $*$ \\
\hline STLF & short term load forecast & \\
\hline DTS / OTS & $\begin{array}{l}\text { dispatcher / operator training } \\
\text { simulator }\end{array}$ & \\
\hline
\end{tabular}

Most of these functions have on-line (real network data connected) and off-line versions (use "frozen" database).

\section{Need for speeding up the calculations}

The classic Load-flow is a precise method for the network modelling but requires huge resources, time, and is also sensible for some parameters.

- Exact (in case of convergence)

- Huge computation requirement

- Used in complex functions (e.g. Contingency analysis) - thousands of LF run is necessary

Sometimes we need an approximate solution to support the fast decision making. The ANN (Artificial Neural Network) seems to be a possible tool:

- Fast calculation

- Parallel operation

- Relatively good solution

The ANN is a general model without the analytical description of the problem. After a specific problem mapping for different application areas the same structure can be applied (mining, pharmacy, biology, signal processing and e.g. power system). By ANN typically two types of the problems can be solved:

- classification (choice from categories)

- retrieval of values of a continuous function

\section{E. The Contingency Analysis (CA) problem}

We know the contingencies (failure, short circuit, outage, line breaking, overload, etc.) but we don't know WHEN and WHERE they happen (like in case of accidents). So, we prepare our network to bear some contingencies. In the (CA) we ask „What happens if one element falls out? Is the power supply continuous and secure?" After checking the fallen out one element, we go on, we restore the net and check the next element till the last. The main steps of the contingency analysis (calculation of security or vulnerability index) are:

- Classification of the different situations - good (secure) / not good (overload, voltage inappropriate, out of supply, etc.)

- $\quad$ N-1 criteria (only one of the elements falls out)

○ step by step over each element

- running a load-flow calculation for each case

- classification

○ $\quad \mathrm{N} * 1$ variation $=\mathrm{N}$ load-flow run

- N-2 criteria

$$
\begin{array}{ll}
\circ & \mathrm{N} *(\mathrm{~N}-1) \text { variation }=\mathrm{N}^{2}-\mathrm{N} \text { run } \\
\circ & \text { Combinatorial explosion }
\end{array}
$$

The off-line CA can work through long days. In case of on-line application e.g. in every $5 \mathrm{~min}$ we should run millions of load-flows to be sure about the security level of the actual network.

\section{F. Error Back Propagation ANN in a $C A$}

The Error Back Propagation ANN is capable of assessing the position of a point if it is inside or outside of a geometrical plane figure (geometrical separation of 
region shapes) [2] (see fig.4.). The two dimensions can be the voltage and the current values. In case of 50 measurements, the problem can be extended into a 50 dimension space (number of dimensions $=$ number of state variables).

\begin{tabular}{|c|c|c|c|c|}
\hline Structure & $\begin{array}{c}\text { Types of } \\
\text { Decision Regions }\end{array}$ & $\begin{array}{c}\text { Exclusive-OR } \\
\text { Problem }\end{array}$ & $\begin{array}{c}\text { Class } \\
\text { Separation }\end{array}$ & $\begin{array}{l}\text { Most General } \\
\text { Region Shapes }\end{array}$ \\
\hline$R^{\text {Single-Layer }}$ & $\begin{array}{l}\text { Half Plane } \\
\text { Bounded By } \\
\text { Hyperplane }\end{array}$ & & & \\
\hline Two-Layer & $\begin{array}{c}\text { Convex Open } \\
\text { Or } \\
\text { Closed Regions }\end{array}$ & & & \\
\hline Three-Layer & $\begin{array}{c}\text { Arbitrary } \\
\text { (Complexity } \\
\text { Limited by No. } \\
\text { of Nodes) }\end{array}$ & & & \\
\hline
\end{tabular}

Fig. 4. Different nonlinear separation problems and BP ANN [2]

The different contingency situations are modelled off-line. The "Good" and "Not good" situations' measurements are taught to the ANN. During the on-line operation the ANN retrieves the classification results quickly.

CA problem mapping -> classification

- State space

- Putting a point into the geometrical space

- Separation into GOOD / NOT GOOD situation

- Classification

\section{G. Neural Nets for short term load forecast}

The scheduling of the power plants operation and managing the normal supply and contingencies are the basic tasks of the system operator. Long term load forecast is required for the system planning but the operation is supported by short term load prognosis (1 day or only some hours ahead).

The load is complicated, the analytical modelling is hardly possible. It is the function of the

$\begin{array}{ll}\text { - } & \text { temperature } \\ \text { - } & \text { wind } \\ \text { - } & \text { the dating } \\ \text { - } & \text { the time } \\ \text { - } & \text { the day type } \\ \text { - } & \text { loads } \\ \text { - } & \text { temperature } \\ \text { - } & \text { season } \\ \text { - } & \text { precipitation, etc. }\end{array}$

The 3 hours ahead utility load forecasts with Quick or Back-Propagation neural net application were accepted tools in the arsenal in the last decades beside the traditional statistical methods.

Similar solutions are used nowadays for the forecast of wind and photovoltaic generation.

Neural Net is also applicable for the hardly modellable tasks as for power market/exchange operation or for dynamic identification and pricing of Available Transfer Capacity at the bottlenecks between countries (ATC). [3]

\section{Metaheuristics}

Sometimes we don't have enough effort or knowledge to find the mathematically perfect solutions but by some heuristic analogy we could reach quite good results. It is used typically for optimization tasks. The characteristics of the 'meta' methods are:

- Complex systems

- Infinite state space

- Optimization (Single or Multi Objective)

- Search in the space

- Quick and tricky

- Relatively good solutions

- $\quad$ The key is the problem representation

The list of the Metaheuristics is long, and these techniques can be applied almost to all technical problems:

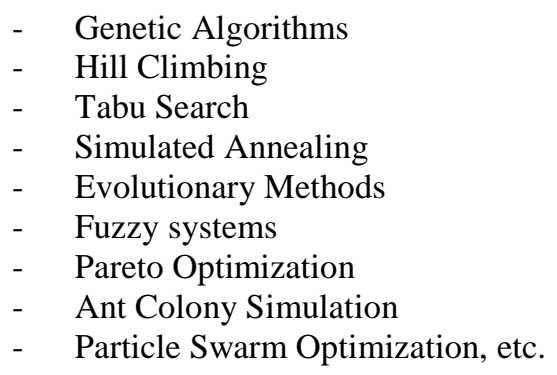

\section{A. Optimum search methods}

A lot of operational problems can be re-conducted to optimum search: smallest, maximal, cheapest, shortest, fastest, etc. The optimum is a point in the space. Different search algorithms have different speed, convergence, stuck-in risk.

- the enumeration (after discretization) scans systematically all the space and gives back the optimal solution. It should take much time.

- the hill climb goes to the steepest direction. It may find local optimum.

- the tabu search wanders in the space but never steps back to the already visited sites

- the simulated annealing looks for the steepest, highest direction but sometimes suddenly changes the direction to avoid the local maxima and stuck-in

- the ant colony search uses the right path that was marked by "pheromone"

On fig. 5. a 3D visualization of the path finding to the highest point of the region can be seen. 


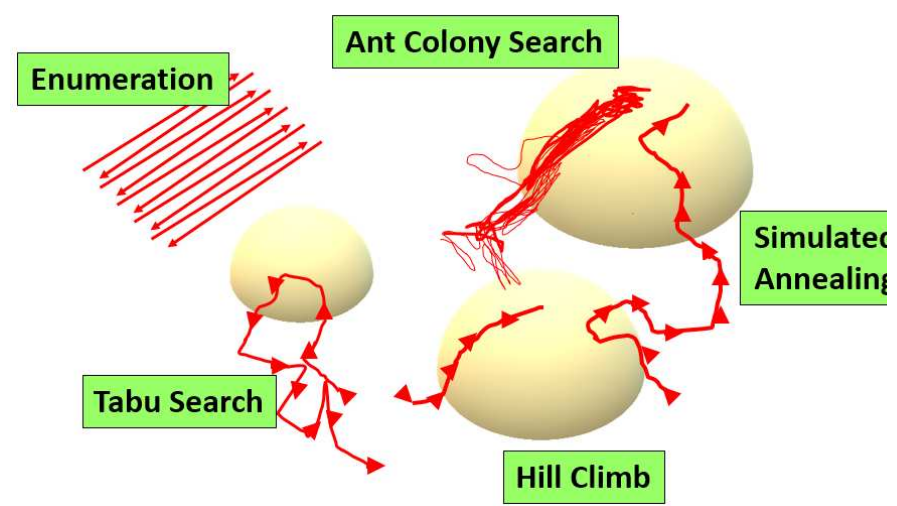

Fig. 5. Metaheuristics optimum search

\section{B. Genetic Algorithms (GA)}

In the genetic representation the main characteristics of the problem are coded to a sequential genome. The actual string is valued (Fitness function). Although combinatorically there are extremely high numbers of the variations, instead of the enumerative technics we use 'metaheuristics'. That means, we make changes on larger parts (mutation). After the evaluation we use only the best specimens for further trials. Main steps:

1 Initialization

2 Selection

3 Operators:

- Crossover

- Mutation

- (Elitism)

4 Breeding

5 Termination

6 Back to selections (2)

\section{Simulated Annealing (SA)}

Physical analogy to SA is taken from metallurgy crystallizing. At low temperature - at low energy level we find the minimum. The temperature decreases slowly. To avoid the possible stuck in to local minimums (as it can happen at Hill climbing method), with some probability we make an ,artificial error”, that is we reheat the system.

\section{Characteristics of Ant Colony Search}

The ants continuously walk, bring and carry materials and information about the visited prospective territories. This path is marked with pheromone [4]. The pheromone level is proportional to the importance of the path. The older pheromones lose their strength, evaporate.

- Search space - system states

- Trail list

- Stronger pheromone update - closer to the minimum

- Dynamic path width

- Multi-objective function

Now we continue with the introduction of some power system related metaheuristics applications.

\section{Problems Solved by Metaheuristics}

\section{A. Transmission system Expansion problem}

Question: WHERE - WHEN - WHICH development is necessary in the network? [5] (see fig.6.)

- Number of possible new connections:

(Nnodes-1)!/2

- Combinatorial explosion

- Cost minimization (construction and loss)

- Simplified / normal Load Flow check

- Voltages in appropriate range

- Currents (line loads) below limit

- Satisfy the transit + load demand

- Possible techniques: Genetic Algorithm Simulated Annealing - Tabu-Search
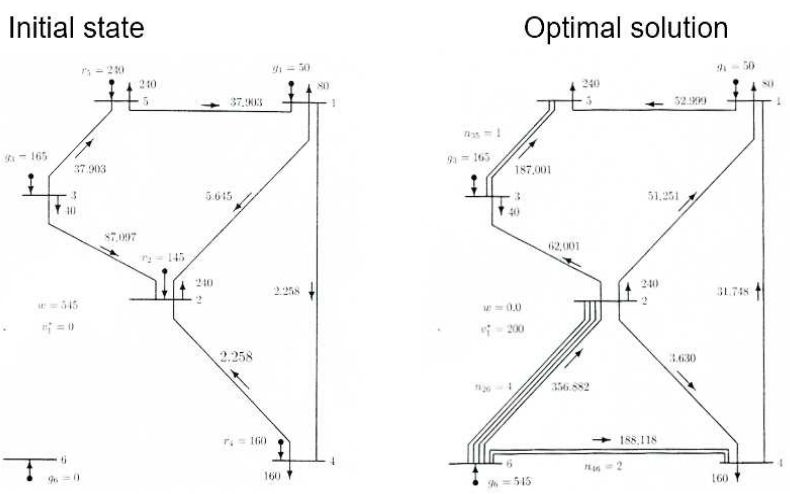

Fig. 6. Transmission system expansion on IEEE 6 bus system

\section{B. Unit commitment / Economic dispatch}

Question: WHERE to generate the required power to minimize the transmission costs?

- Objective function - minimum cost

- Power constraint

- Different generation cost functions

- Unit constraint

- Dozens of units

- Possible techniques: Genetic Algorithm + network loss calculation by LoadFlow

C. Optimal Scheduling (Economic dispatch), reserve allocation

Problem: Economic dispatch in consecutive steps in time takes into account the cost at a Mode of Operation. [6]

- We minimize the total generation cost including constant and fuel-dependent components, and reserve allocation costs as well. We consider positive and negative secondary and tertiary reserves.

- Cost at a Mode of Operation:

$\mathrm{MoO}$ cost $=$ generation cost + reserve allocation + changeover costs

$$
C(\mathrm{f})=C G(\mathrm{f})+C R(\mathrm{f})+C C O(\mathrm{f})
$$


- Objective function: minimize $\sum C(f)$ at several constraints

- Nonconvex quadratic optimization

- Optimal power dispatch based on linear decomposition (see fig.7.)

- Possible technique: MATLAB solver

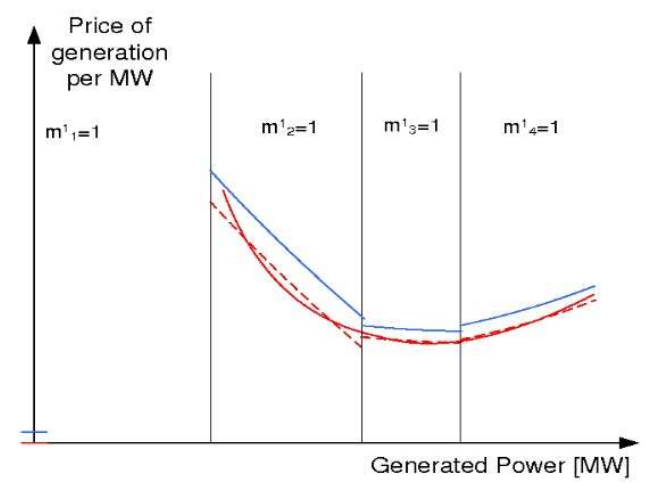

Fig. 7. Unit generation cost curve

\section{Scheduling of multi-cogeneration system}

Problem: In the distributed heat and power cogeneration system the generation must be scheduled by heat demand, taking into account the heat storage tanks' status and the power selling possibilities. [7]

Minimize the buying cost of electricity + fuel cost of cogeneration + auxiliary boilers + independent generators

- Possible technique: Genetic Algorithm

\section{E. Constrained Load Flow problem (reactive power balance)}

Looking for the minimum loss situation by taking into account the ampacity, reactive power and voltage constraints.

- „Traditional” problem (60 years old)

- „Welfare” of the network, the power flows are slightly influenced

- Influencing points in the substations (in the nodes see fig. 8.) are

- Transformer tap positioning (discrete positions)

- Synchronous compensators (continuous range)

- Generator excitation (continuous range)

- Many devices, too many possible settings combinatorial explosion

- Possible technique: Ant Colony Search

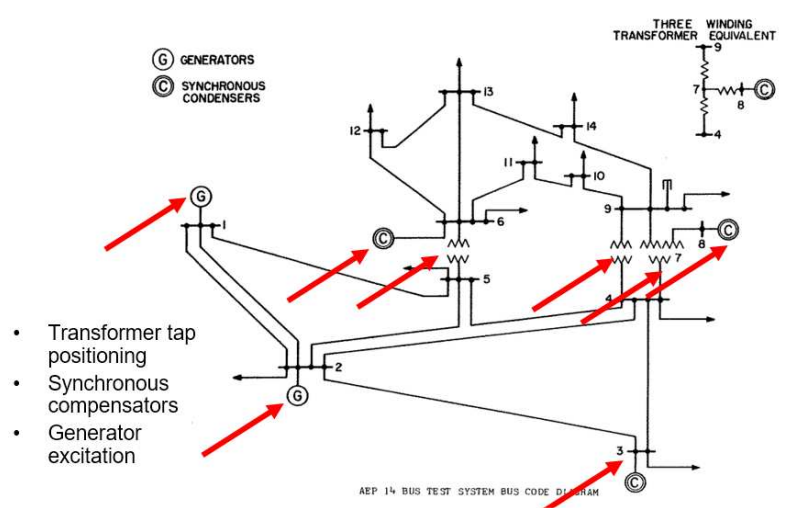

Fig. 8. CLP influencing points

\section{F. Algorithms for Power Flow Control devices}

It is similar to the previous problem but more control devices are settled on the lines with continuous control range. [8]

- „New” problem (15 years old)

- Flow influencing points are on the lines (see fig.9.)

- FACT - Flexible AC Transmission devices

- Power flow controller

- Series compensator

- HVDC line

- Power electronics

- Continuous control range - discretization

- Possible technique: Ant Colony Search

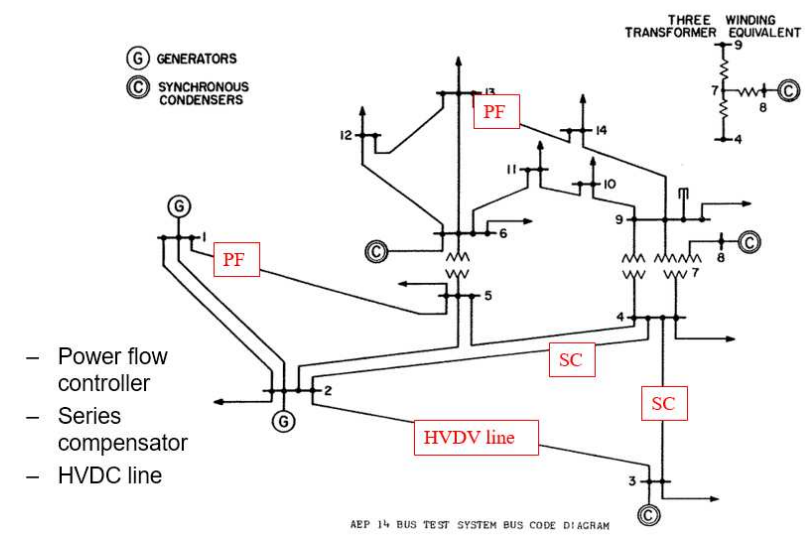

Fig. 9. OPF influencing points

\section{G. Power mix optimization}

We are looking for an optimal extension to the present power generation mix (coal, gas, hydro, PV, wind, nuclear, etc.) [9]

- Single Objective Optimization

- Looking for the appropriate energy mix portfolio with:

- $\mathrm{CO} 2$ emission minimization OR

- Externality minimization OR

- Investment minimization

- AND - more objectives simultaneously - MOO (Multi Objective Optimization)

- Possible technique: Linear Programming

On fig. 10. one can see the optimal generation portfolios. 


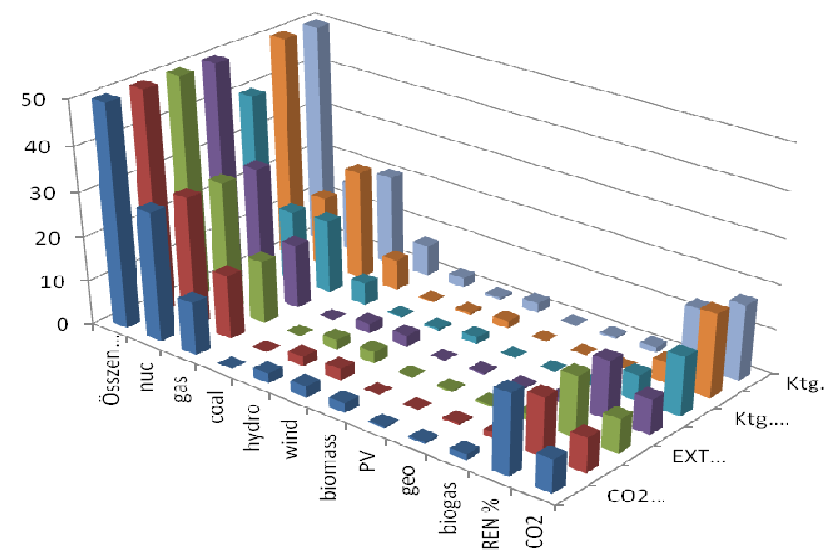

Fig. 10. Generation ratios in different optimization alternatives

\section{H. Trading with energy}

The non-physical trade on the power exchange looks for the optimal (cheapest) trading path (see fig. 11.). As constraint one must handle the inter-state bottle necks. [10]

- trade-flow modelling (not real power flow!)

- demand - offer balance

- price compensation mechanism

- market-coupling

- cross border capacity allocation

- individual transactions

- optimization for bottle neck flow - different interests of trader (cost minimization) and system operator (system stability)

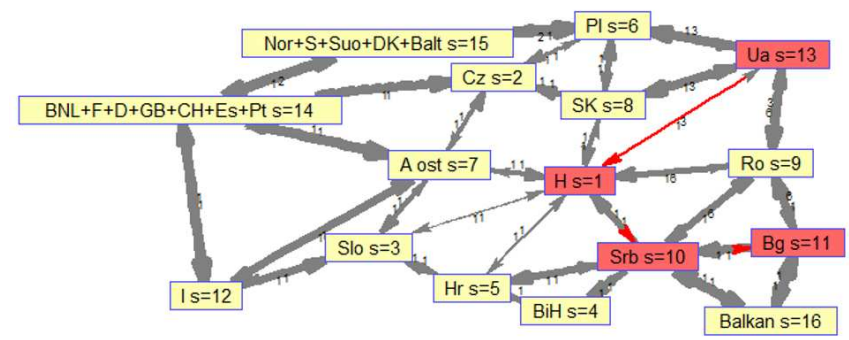

Fig. 11. Cheapest path

The main idea of the simulation is that we are looking for the optimal - that is the minimal price. All the countries are looking for the appropriate amount of energy at the cheapest price. In the first step we minimize the total market costs (the sum of all national energy costs) - Single Objective Optimization.

- Possible technique: Linear Programming

\section{Conclusion}

The world itself and the power systems are getting more complex. The efficient control requires metaheuristic approaches. Network analysis becomes on-line (like the fuzzy technique in the industry). The problem of the Multi Object Optimization has a human factor, somebody should decide what the real objective is. The optimal solutions can be calculated - but it is not at all easy to put them into action in reality.

\section{References}

[1] Rich Christie, "Power Systems Test Case Archive," Aug. 1993, 「Accessed Feb. 4, 20181. 「Onlinel. Available: http://www.ee.washington.edu/research/pstca/pf14/pg_tca1 4bus.htm

[2] R P Lippman. An introduction to computing with neural nets. IEEE ASSP Magazine, 4(2):4-22, April 1987

[3] P. Kádár - Zs. Bertalan: Neural Net Supports the utility in the deregulated power system; Power Quality'98 conference; Santa Clara, California, USA, November 1012, 1998, pp.351-359

[4] L. Kwang et al.: Constrained Load Flow problem in Modern Heuristic Optimisation Techniques

[5] E. Asada et al.: Transmission network expansion in Modern Heuristic Optimisation Techniques

[6] D. Csercsik and P. Kádár, „Performance analysis of MATLAB solvers in the case of a quadratic programming generation scheduling optimization problem" Proceedings of the 19th International Conference on Power Electronics and Power Systems, May 25-26, London, England, 2017, pp. 3485-3489, EISSN: 2010-3778

[7] L. Kwang et al: : Cogeneration scheduling in Modern Heuristic Optimisation Techniques

[8] L. Lai - N. Sinha: OPF with FACT devices in Modern Heuristic Optimisation Techniques

[9] P. Kadar: Multi Objective Power Mix Optimization; SAMI 2010 Herl'any, SK Jan. 29-31. 2010

[10] P. Kadar: Regional power trade modeling; ISAP 2011, Crete, Greece - 26-28. Sept. 2011 